\title{
Automated Screening of 2D Crystallization Trials Using Transmission Electron Microscopy: A High-Throughput Tool-Chain for Sample Preparation and Microscopic Analysis
}

\author{
Nicolas Coudray ${ }^{\text {a }}$, Gilles Hermann ${ }^{\text {a }}$, Daniel Caujolle-Bert , Argyro Karathanou ${ }^{\text {, }}$, Françoise Erne- \\ Brand , Jean-Luc Buessler , Pamela Daum ", Juergen Plitzko , Mohamed Chami , Urs Mueller , \\ Hubert Kihl , Jean-Philippe Urban , Andreas Engel and Hervé-W. Rémigy \\ a \\ MIPS laboratory, University of Haute Alsace, 4 rue des Frères Lumière, 68093 Mulhouse, \\ France \\ b \\ C-CINA - M.E. Mueller Institute for Structural Biology, Biozentrum, University of Basel, \\ Switzerland \\ $\mathrm{c}$ \\ Max Planck Institute of Biochemistry, Martinsried, Germany \\ d \\ Department of Pharmacology, Case Western Reserve University, Cleveland, OH, USA \\ FEI Electron Optics BV, Eindhoven, The Netherlands \\ *corresponding author: Dr. Hervé-William Rémigy \\ FEI Electron Optics BV, Eindhoven, The Netherlands
}

\begin{abstract}
Abbreviations
$\mathrm{C}_{\mathrm{ROI}}$, Circle delimiting the Regions Of Interest; C8E4, Octyltetraoxyethylene; DDM, Dodecyl Maltoside; DMPC, Dimyristoylphosphatidylcholine; DM, Decyl Maltoside; GUI, Graphic User Interface; HEPES, 4-(2-hydroxyethyl)-1-piperazineethanesulfonic acid; HTML, HyperText Mark-up Language; MBCD, methyl beta cyclodextrin; Octyl-POE, octyl-polyoxyethylene; PED, Partial Edge Detection; ROI, Region of Interest; $\mathrm{R}_{\mathrm{rc}}, \mathrm{C}_{\mathrm{ROI}}$ Circle Radius; TEM, transmission electron microscope;
\end{abstract}




\begin{abstract}
We have built and extensively tested a tool-chain to prepare and screen two-dimensional crystals of membrane proteins by transmission electron microscopy (TEM) at room temperature. This automated process is an extension of a new procedure described recently that allows membrane protein 2D crystallization in parallel (Iacovache et al., 2010). The system includes a gantry robot that transfers and prepares the crystalline solutions on grids suitable for TEM analysis and an entirely automated microscope that can analyze 96 grids at once without human interference. The operation of the system at the user level is solely controlled within the MATLAB environment: the commands to perform sample handling (loading/unloading in the microscope), microscope steering (magnification, focus, image acquisition etc.) as well as automatic crystal detection have been implemented. Different types of thin samples can efficiently be screened provided that the particular detection algorithm is adapted to the specific task. Hence, operating time can be shared between multiple users. This is a major step towards the integration of transmission electron microscopy into a high throughput work-flow.
\end{abstract}

\title{
Keywords
}

Automation; 2D crystallization; electron crystallography; grid preparation; high throughput; image processing; membrane protein; negative staining. 


\section{Introduction}

Membrane proteins are essential for cellular life, but structural information on membrane proteins is sparse, because most of them do not form 3D crystals. Reconstitution of membrane proteins into the lipidic bilayer can yield under specific conditions two-dimensional crystals suitable for high-resolution analysis. To identify such conditions is a tedious work where each step is critical. Automation of repetitive manipulations not only improves screening speed but also enhances reproducibility and thus enables biologists to focus on critical tasks such as protein expression and purification. As result of the small size of 2D crystals (thickness down to less than $10 \mathrm{~nm}$ ), sample deposition on electron microscopy grids covered with a carbon film, negative staining, and electron microscope analysis are required to monitor the screening process. Several approaches have been introduced previously (Vink et al., 2007; Cheng et al., 2007). Our effort aims at setting up a high throughput screening process for $2 \mathrm{D}$ crystallization of membrane proteins similar to methods used in 3D crystallization since many years, and is motivated by the recent advances in two dimensional crystallography (Gonen et al., 2005, Fujiyoshi et al., 2008).

Manufacturers have been developing a class of transmission electron microscopes (TEMs) and related instruments to meet cellular and structural biology needs (FEI Company, Gatan Inc., Jeol Limited). Among other new features (helium cooled stages, dual tilt axis, double coiled lenses, nitrogen auto-filling, microscope remote control etc.) systems handling multiple grids have been designed. The grid auto-loading technology opened up new avenues in sample screenings. In the setup described here, the FEI Autoloader solution has been used: after each sample has been deposited on an EM grid, washed and stained using a staining robot, each grid is placed on a small ring compatible with the microscope stage and inserted in a cassette. Each cassette can host up to 12 grids. An Autoloader coupled to the microscope picks up the cassette automatically from a "Carousel". This Carousel can host up to 8 cassettes for a total of 96 grids. Once in the Autoloader, the cassette and its 12 grids remain in vacuum, enabling for fast sample loading into the column of the microscope. A server hosted on the microscope's computer is waiting for commands coming from the MATLAB environment installed on a remote computer, which controls the screening process. This system has been used to reconstitute and crystallize several proteins during its development. Beside the bacterial porin OmpF routinely tested, we crystallized a new type of membrane protein which is a complex made of the hemophore HasA and its receptor HasR, an outer membrane protein from Serratia marcescens.

\section{Material and methods}

\subsection{Staining robot}

Grids and Grid holder. 200 mesh nickel grids (Quantifoil) were used for screening. Nickel grids with others meshes are available as well. The grid holder base is made of stainless steel. Under each nickel grid a small magnet is immobilized to keep the grid in place (see Fig. S4, Supplementary Materials). A berylium-copper alloy sheet on which lays a paraffin film (Parafilm PM996, Pechiney, Chicago) covers the magnets. A grid index designating the samples is engraved in this sheet. This holder is a 1:2 scale replica of commercially available 96 well microplates. Hence the pitch between the center of the grids is $4.5 \mathrm{~mm}$ in both $\mathrm{X}$ and $\mathrm{Y}$-axis for 
the grid holder, instead of $9 \mathrm{~mm}$ for a standard microplate. Above the paraffin film a mask also made of annealed berylium-copper alloy is fixed to insure rigidity, and to center the 96 grids during deposition by the operator. The magnets help to pull the nickel grids away from the operator's tweezers and facilitate their deposition. To keep the mask in place during the grid deposition procedure, carbon evaporation and glow discharging, 4 magnets were glued on both sides of it and correspondingly, 4 magnets were embedded into the grid holder.

Plastic film deposit. The holder with the grids in place is immersed in a large beaker filled with distilled water. A droplet of $2 \%$ parlodion in N-butyl acetate is deposited on the surface of the water using a Pasteur glass pipette and left to dry for 2 minutes. The water level is then lowered using a valve located at the bottom of the beaker until the plastic deposits on the surface of the paraffin film. The holder is then taken out of the beaker and dried overnight at room temperature.

Carbon evaporation and glow discharging. The mask initially present during the grid deposit on the paraffin film is put back for the carbon evaporation procedure. It will prevent carbon to be evaporated beyond the grid perimeter and thus avoid sample spreading beyond. After carbon coating, the mask is left in the grid holder during glow discharging at low pressure in air in a home built system (Aebi et al. 1987). This procedure is performed to render the grid area hydrophilic.

Staining procedure. The 12 microplate's rows containing 8 samples from the $2 \mathrm{D}$-crystallization robot are processed one after the other. Because the robot's pipettes have a spacing of $9 \mathrm{~mm}$, but the grids a pitch of $4.5 \mathrm{~mm}, 4$ sample drops of one row, containing $4 \mu \mathrm{l}$ each, are pipetted out from the microplate and deposited on grids 1,3,5 and 7. While the samples are incubated for 60 seconds, the pipettes are washed with water in a sonifying bath. Subsequently, $4 \mu 1$ of water are dispensed on each of the four grids and immediately sucked away. The water washing cycle is performed 3 times for 2D crystals obtained by dialysis and 9 times for 2D crystals obtained with the cyclodextrin method. Pipettes are washed again. Then $3 \mu 1$ of a $2 \%$ uranyl acetate solution are pipetted from another microplate, deposited on the four grids, incubated for 15 seconds, and sucked away into a waste microplate that is disposed according to safety regulations. The pipettes are purged to avoid contamination with remaining radioactive staining, and finally washed extensively in the sonifying bath. This process is then repeated with grid 2,4,6 and 8 . Grids are finally left to dry at room temperature for few minutes.

The staining interface (see Fig. S5, Supplementary Materials) allows all the parameters (volumes, times, number of washing and staining cycles) to be varied, and prepared sample to be selected. It also enables mixing and dilution in the 96-microplate if this was required.

Staining robot, pipetting device and electron microscopy. The staining robot has been built on a Nano25 gantry robot from Hudson Control Group (New Jersey, USA). The 8 channels pipettor is from Seyonic S.A. (Neuchatel, Switzerland). Electron micrographs of negatively stained specimens have been acquired using a Tecnai-12 operated at $100 \mathrm{kV}$ (FEI electron optics, Eindhoven, the Netherlands) equipped with a $1 \mathrm{k}$ x $1 \mathrm{k}$ pixels CCD camera, an Autoloader and a Carousel, using magnifications x60, x 1350 and $\times 21 \mathrm{k}$ corresponding to 299, 12.9, and 0.813 $\mathrm{nm} /$ pixel respectively.

\subsection{High-throughput screening}


The screening system is made of (i) a T12 microscope equipped with an Autoloader (Fig. 1), (ii) a "microscope computer" hosting the servers dedicated to command the microscope and the Autoloader, and exchanging data with the "image processing computer", and (iii) an "image processing computer", sending commands to the "microscope computer" to perform the acquisition scheme, and extracting data from the images. The "microscope computer" runs a HTML server receiving commands sent by the "image processing computer". The "image processing computer" runs "ANIMATED-TEM" (Coudray et al., 2007) for controlling the screening strategy and performing image analysis in real time. As The MATLAB software controlling the screening session hosted on a separate computer is entirely independent from the microscope hardware, it can be adapted to any automatic screening configuration or standard microscope. The servers hosted on the microscope computer were developed specifically for the hardware (i.e. Autoloader, Carousel) presented in this tool-chain.

\subsubsection{Automated sample exchange}

The control interface hosted by the "microscope computer" steers the Autoloader and the Carousel through their respective server. The Autoloader loads and unloads grids into the microscope from a "Cassette" containing up to 12 mini-cartridges also called "Autogrids". An Autogrid is an assembly made of a ring, a circular shaped clip ("Circlip") and a $3 \mathrm{~mm}$ standard EM grid. We used Circlips made of a Titanium/Nickel alloy (Memry GmbH, Weil am Rhein, Germany). When a Cassette is docked into the Autoloader, air is pumped out and the 12 grids remain in a high vacuum during screening. The Autoloader needs to be vented again only after the screening of the 12 grids has been completed. This allows for rapid sample exchange. After a Cassette has been undocked, the Carousel provides the next one. The Carousel has a gun revolver configuration hosting 8 cassettes. The Carousel control is executed from MATLAB via the "microscope computer's" control interface through HTTP requests.

\subsubsection{Image processing framework}

The "image processing computer" is equipped with an image processing tools suite, known as ANIMATED-TEM (Analysis of Image for Automatic Targeting and Extraction of Data in Transmission Electron Microscopy (paper in preparation) and developed in the MATLAB environment. Its goal is twofold: to control the acquisition scheme, and to extract data for the sample characterization. Therefore, the selection of targets by image processing algorithms will lead to a fully automatic acquisition of images for specimen assessment.

The acquisition scheme is organized in three steps: images acquired at low magnification (x60) are automatically analyzed to identify convenient grid squares and to separate those with damaged carbon films. Then, the coordinates of the selected grid squares are sent to the "microscope computer" for acquisitions at medium magnification (x1350). Images acquired at medium magnification are then processed to select potentially crystalline areas. Images of the selected proteoliposomes and crystalline sheets are finally acquired at high magnification (x21000) by the "microscope computer".

The data automatically extracted at the different magnifications, allow the characterization (i) of the grid, i.e. amount of damaged grid squares (at low magnification), (ii) of the membrane, i.e. 
their size, stacking, and shape (at medium magnification), and (iii) of their crystallinity through the analysis of the diffraction patterns (at high magnification). The principles of the algorithms (Karathanou et al. 2010) are summarized below.

\section{Low Magnification analysis - grid quality assessment}

After a global thresholding of the image, the histogram of each grid square is computed (Fig. 2A) and independently segmented to identify the gray-level of the backgrounds, i.e. the last peak of the histograms. An analysis of the backgrounds and their gray-level is used to determine whether the carbon film is locally broken or not. Selected squares correspond to non-broken zones (Fig. 2B).

\section{Medium Magnification analysis - Membrane characterization}

At medium magnification, image analysis is especially difficult because the wide variety of object aspects that could be observed after a 2D crystallization experiment. The algorithms developed can select ROIs (Region of Interest) and trigger high magnification acquisitions, and statistical features to identify proteoliposomes can be extracted. However, in some cases of low sample quality (especially when the staining quality is poor), the statistics can be biased and the efficiency of the selections could be affected. A global histogram classification (Coudray et al., 2007) of the acquired image is applied to identify unsuitable areas containing severe stacking or contaminations. Images of membranes are processed using a set of algorithms (Fig. 3): first, images are split into homogeneous regions using a multi-resolution edge-based algorithm (Fig. 3B); second, based on a statistical validation of the transitions, false edges are removed using merging (Fig. 3C); third, each region is semantically labeled according to its potential stacking (Fig.3D).

Finally, for the selection of the ROIs, the user can choose the method and the maximum amount of ROIs per image. The first selection option, called the Partial Edge Detection (PED) method, is a fast semi-random selection positioning ROIs near edges found using a typical edge detector. The second option is based on the characterization described above, and selects ROIs among the non-stacked regions, relying on criteria such as size and shape.

\section{High Magnification analysis - Assessment of crystallinity}

At high magnification (Fig. 4), before diffraction spots detection, the background of the power spectra of the image is evaluated using a mean radial transform in order to remove the effect of the Contrast Transfer Function. After background correction, spots with signal to noise ratio above 3.5 are selected.

\section{Scenario of a typical screening session}

The scenario of a fully automated session is as follows: first, an image is acquired at the center of the grid at low magnification. When no square is selected in the current field of view, the stage is moved to another position according to a spiral pattern in order to minimize the displacements. Second, if a sufficient number of grid squares are selected, a medium magnification image is acquired at the first targeted square and analyzed to identify ROIs. If no ROI is identified, another medium magnification image is acquired at the next selected grid square. Third, if ROIs have been selected, high magnification images are acquired for each selection and the process 
goes back to the next grid square at medium magnification, or acquires a new low magnification image.

To setup a scenario before the screening session is initiated, the user selects parameters in a GUI (Fig. 5), which define how images are acquired (magnification, exposure time, illumination, frequency of the autofocusing) and when the process should stop (maximal amount of images to acquire).

Acquisition. Default parameters proven to be effective in general are used to acquire micrographs at low magnification (x60), medium magnification (x1350) and high magnification (x21000). These parameters depend on the specific equipment (mostly on the CCD). For each magnification the illumination, the exposure time and the defocus can be set. The user has the possibility to do a sanity check of the stage behavior. If needed a stage calibration can be performed.

Control and image processing. The number of images per grid square and the number of images per ROI and the frequency of the focus measurement are selected ( 1 measure per ROI in each case by default). The maximum number of ROIs to be detected by each possible detection method (the PED and the Multi-Resolution method) is chosen. Diffraction spots detection is enabled by default. To limit the screening period per grid the maximum amount of images required to identify grid square of good quality, and the maximum number of grid squares to be visited are set by the operator.

Advanced parameters. For each magnification, advanced parameters can be modified: for low magnification, to qualify a grid square as a good one, a minimal area corresponding to a given percentage of the largest grid square area previously detected can be set; for medium magnification, a minimal and a maximum expected ROI diameter can be adjusted. Also the target selection priority (large regions, regions with linear edges, contrasted region, regions with representative gray-level) can be ranked; for high magnification, the diffraction spots detection thresholds and the resolution range (from $1 / 2$ to $1 / 16 \mathrm{~nm}^{-1}$ by default) can be set. Finally, an interactive matrix depicting the 8 cassettes and their 12 respective grids enable the operator to select which grids should be analyzed (Fig. S6, Supplementary Materials).

\subsection{Purification and crystallization}

\subsubsection{OmpF}

The bacterial outer membrane OmpF was produced in E.coli strain BZB1110/pMY222 and purified in Octyl-POE as reported previously (Holzenburg et al., 1989). The initial OmpF concentration ranged from 1 to $2 \mathrm{mg} / \mathrm{ml}$ in various buffers. DMPC, E.coli lipids and a variety of phospholipids mixtures were used for reconstitution and the lipid to protein ratio ranged from 0.2 to 0.8. OmpF was reconstituted using the 2DX Robot (Iacovache et al., 2010). After reconstitution, the 96 wells microplate was transferred in the staining robot. After staining each EM grid is mounted in its respective Autogrid, placed in Cassettes, and finally inserted into the carousel, ready to be screened by TEM. OmpF crystallization conditions are well known, but we decided deliberately to use wide screening conditions to test our system. Ternary mixtures (lipid/protein/detergent) were prepared automatically with the corresponding buffers using the 
“Ternary Mixture Robot” (See Fig. S3, Supplementary Materials), a customized pipetting system based on the Precision XS robot (Biotek, Vermont, USA).

\subsubsection{HasA-HasR-heme complex}

HasA-HasR-heme complex (Krieg et al., 2009) was purified in octyltetraoxyethylene (C8E4) and the detergent was exchanged with Dodecyl Maltoside (DDM) before 2D-crystallization. Purified complex was mixed with different lipids to obtain a matrix of 96 conditions, and placed on a standard 96 wells transparent plate. This plate was processed for screening in the same way as the OmpF plate described above. The same crystallization buffers used for OmpF were used for HasA-HasR-heme complex.

\section{Results and discussion}

Recent advances of TEM technologies opened avenues for the development of new methods. Tomography (Tom tool box (Nickell et al., 2005), Cemovis (Al-amoudi et al., 2004)) and single particle analysis (Cong et al., 2010) are taking advantage of improved computational power and the availability of high performance electron microscopes, which have been equipped for cryoelectron microscopy of biological samples (Dubochet et al., 1981). Outstanding results (acetylcholine receptor at $4.0 \AA$ (Unwin et al., 2005), Gluthathione transferase at $3.2 \AA$ (Holm et al., 2006), AQP4 at $2.8 \AA$ (Tani et al., 2009), and AQP0 at 1.9 $($ Gonen et al., 2005)) show that electron crystallography gained a new momentum (Hite et al., 2007), and justify our efforts to set up 2D crystallization screens similar to those available for 3D crystallization.

\subsection{Membrane protein reconstitution and high-throughput}

The first and most fundamental challenge in determination of membrane protein structures by electron crystallography is to express and purify the membrane proteins. The next one is to obtain two-dimensional crystals. These two steps are closely related since the nature of the detergent used for the protein extraction from the cell membrane will influence the reconstitution of the protein in the lipid bilayer during crystallization (Hasler et al., 1998, Rigaud et al., 2000). However, every membrane protein has its own behavior and no universal isolation recipe is available. For example, Triton TX-100 was used during the isolation of microsomal Gluthathione transferase (Morgenstern et al., 2005), and purification of AQP0 required Decyl Maltoside (Gonen et al., 2004). Only empirical approaches were successful until now making large screening of crystallization conditions a necessity. Several methods have been developed to perform 2D crystallization: dialysis against detergent-free buffer (Jap et al., 1992), adsorption of detergent molecules to polystyrene beads (Rigaud et al., 1997) dilution of crystallization mixture below the critical micelle concentration (CMC) of the detergent (Rémigy et al., 2003), and the use of cyclodextrins to chelate the detergent in solution (Signorell et al., 2007). Based on these approaches several devices have been engineered to improve 2D crystallization throughput: dialysis (Ringler et al., 2000; Vink et al., 2007), polystyrene beads (Nakata and Inoue, 1998) and cyclodextrin (Iacovache et al., 2010). As the cyclodextrin method allows the kinetics to be 
accurately controlled by quantitative addition of MBCD using nano-fluidic dispensers in parallel, this has been the method selected for our tool-chain.

\subsection{Automatic negative staining}

Negative staining is a method consisting of the deposition of heavy atoms salts that will surround the protuberances of the sample revealing its topology (Hayat et al., 1990). Uranyl acetate, uranyl formate, phosphotungstic acid (PTA), and osmium tetroxyde are stable under the electron beam and have been widely used for decades for contrasting of cells, single particles, and 2D crystals for electron microscopic analysis at room temperature. We used uranyl acetate in our automatic negative staining procedure as it is less prone to precipitation than uranyl formate, and it provides excellent contrast routinely.

Since 2D crystals are very thin samples screening cannot be done using light microscopes like in 3D X-ray crystallography. Only EM provides enough resolution to visualize the 5 to 12 nanometers thick crystalline membranes. The monitoring of such thin specimen in an EM requires their deposition on electron microscopy grids that have a diameter of $3 \mathrm{~mm}$ and are made of metal covered by a carbon layer.

Grid preparation. Nickel grids were used in our experiments. The most common procedure to prepare such carbon-coated grid is to spread carbon by evaporation on a mica foil (Harris et al., 1997). While this mica approach is common practice, it cannot be used to cover such large surfaces as taken by 96 grids (about $4 \times 6 \mathrm{~cm}$ ). Therefore, a parlodion film is deposited on the grid before carbon evaporation to strengthen the carbon layer, although free detergent remaining in the sample after reconstitution may destroy it. Crystalline samples are generally detergent-free, but traces of detergent may remain. If the film is severally broken, the amount of detergent in the sample should be measured (Kaufmann et al., 2006), and detergent removal needs to be completed before grid preparation.

Depositing grids on the plastic film requires skill and is cumbersome. Using our grid holder, the combination of a grid mask and small magnets makes this tedious work much easier and insures that the $4.5 \mathrm{~mm}$ distance between grids is constant over the entire plate. No further individual handling of any grid is necessary until they are inserted into the microscope. We have tested grid holder prototypes where the grids were mechanically clamped, but these showed to introduce unevenness. The use of magnets combined with nickel grids solved this problem.

Carbon evaporation and glow discharging. It was not possible to have an evenly spread parlodion film that covers a full size $(128 \times 86 \mathrm{~mm}) 96$ grid holder compatible with the 96 well microplate. Also, our glow discharger could not accommodate such a large grid holder. Our grid holder (also referenced as a grid block by Vink et al., (2007)) being the quarter of the size of a standard microplate, fitted into our home built glow discharger.

Grid manipulation during sample deposit / washing / staining. Cheng et al. (2007) have addressed the development of a mechanism that clamps the grids down. The magnetic field between the nickel grids and the small magnets embedded in the staining block is strong enough to keep the grid in place during the pipetting process. Our first attempts to prepare samples were made using a Teflon block, this material being hydrophobic. Nevertheless it did not prevent grid 
contamination due to liquid spreading by capillary force, a crucial issue when one wants to screen samples using staining like uranyl acetate. The hydrophobic fence built up by the combination of paraffin film and the use of the grid mask during glow discharging prevented spreading of sample away from the grid and permitted a complete recovery of the staining liquid. Parafilm has a surface with well-defined properties (Kaufmann et al., 2006), is commercially available, and can safely be disposed after exposure to uranyl acetate to meet regulations about radioactive substances. Since the mask is removed after glow discharging, sample or staining touch nothing else than the grid or eventually the paraffin film. Hence the grid holder and the mask can directly be used in a next round without further cleaning.

Micrograph quality. We have been afraid of image distortions during electron micrograph acquisitions because of the ferromagnetic nature of the nickel grids, in particular at low magnification. Surprisingly, as shown in Fig. 2-4, 6, and 7 comparable results as with the copper grids were obtained for each magnification.

Pipettors technology. A multiple channels dispenser is required especially when extensive washing with water is required. The 8 channels pipettor took about 50 minutes to prepare 96 samples depending on the chosen conditions (incubation times, number of washing cycles). To prevent lag of time between washing and staining, 4 samples (half a column) are entirely processed at a time. A 96 channels pipettor like the Biomek FX liquid-handling robot as used by Vink et al. (2007) does not need pipette washing steps, but raises the cost of equipment and disposable tips. The recycling of disposable tips should not be forgotten when working with uranium salts. The inline dispensing ability of the 8 Seyonics tips enable for fast and extensive washing of cyclodextrin when this compound has been used to neutralize detergent during membrane protein crystallization.

\subsection{Electron microscopy and sample exchange}

The need for automated screenings is underlined by the recent efforts done by multiple groups to improve steps mentioned above (Carragher et al., 2000; Fellmann et al., 2002; Potter et al., 2004; Suloway et al., 2005; Vink et al., 2007; and Cheng et al., 2007). The "Gatling Gun" (Lefman et al., 2007) can host up to 100 grids fastened on as many electron microscope holder tips. Our approach is based on loading 12 grids each mounted in a special ring, fastened by a circlip and inserted in a cassette. The circlip is made of a special titanium-nickel alloy (Nitinol) having a memory effect. At room temperature the diameter of the circlip is larger than at $0^{\circ} \mathrm{C}$. Upon plunging of the ring-grid-circlip assembly in liquid nitrogen, the circlip diameter decreases dramatically, releasing the grid from the ring. The ring and the circlip can then be reused. 8 cassettes are loaded in the Carousel for a total of 96 grids. During the screening process, each cassette will be docked into the Autoloader in a high vacuum environment for 12 grids to be ready for loading in the microscope. Hence the vacuum is established only once during cassette docking, which takes 6 minutes, and not each time a grid is loaded as with conventional microscopes. Because a typical venting/pumping cycle required for loading a grid takes around 2 to 3 minutes, the time gained during the screening of 96 grids is decisive in a high-throughput strategy.

\subsection{Sample screening strategy}


Intensive screenings including automatic grid loading and unloading have been successfully performed. Typical results obtained with a fully loaded Carousel (96 grids) are described below. Such a screen can be accomplished within 36-60 h, depending on the screening strategy.

\section{Screening method principles}

To detect grid squares of good quality, a global and a local background thresholding is performed on images taken at low magnification, followed by a statistical analysis of the local shapes and the gray-levels (Fig. 2). The coordinates of the selected squares are used to acquire medium magnification images. The algorithm takes about 2 seconds to process a $1024 \times 1024$ pixel image. Table S1 (Supplementary Materials) shows the success rate of automatic selection to be $>80 \%$ of the manual selection for selected grid squares and $>99 \%$ for rejected ones. To improve throughput mapping of the entire grid is not performed: if no square is selected in the acquired field of view, the stage is moved to another position.

When an image is analyzed and the stage is immediately moved to the desired target, the imprecision is sufficiently low (a few $\mu \mathrm{m}$ maximum at low magnification) to allow a good targeting. However, when several targets are selected on a single image, the move to the last target is also biased by the addition of the imprecisions of the previous displacements. At low magnification, when small grid squares are used (400 mesh grids), the increasing imprecision is such that after the 4th consecutive grid square, the target reached encloses a too important part of the copper grid bars to be analyzed at medium magnification. It would be possible to add an algorithm based on correlation that would adjust the position, but it would increase the computation-time. A simpler and faster solution is to use larger mesh grids to avoid the risk of targeting the copper bars. In our cases, 200 mesh grids were the most suitable.

After detection of ROIs at medium magnification, the microscope is set to high magnification. An autofocusing is executed before each acquisition. Autofocus was done using the autocorrelation method, but other approaches are currently under investigation. An image of a ROI is acquired and its power spectrum calculated. No mask is applied during Fourier transformation. The diffraction peak detection is performed in a 2-step method that does not require any a priori knowledge of the lattice, of the CTF or of the microscope setup (Coudray et al., 2007): to correct for the CTF contribution, the mean radial profile (Fig. 4C) is calculated by rotational averaging of the power spectrum (Mallick et al., 2005) and subtracted from the power spectra image. The resulting image is then segmented by thresholding (Fig. 4D), sorting Fourier transforms with and without diffraction peaks with a success rate above $99 \%$.

\subsection{Automatic run timing}

After each run, a table summarizing the screening performance is recorded. The time required to analyze a grid depends on two parameters: first, the initial settings of the run, i.e. the maximal number of images to be acquired at each magnification. Second, the content of the grid: for instance, when the grid shows too many broken squares, only low magnification images are acquired and analyzed. It also shows the number of times each step has been performed and the time spent during each step. A typical 96-grids run including OmpF and HasA-HasR-heme screening has been achieved in 54 hours and 20 minutes (Table 1) during which 6014 images have been acquired (1651 at low magnification, 1351 at medium magnification and 3012 at high 
magnification). Time per grid varied from 8 minutes to 89 minutes. Autofocusing performed before high magnification image acquisition was the most time-consuming step (53.7\%). 28 grids have shown diffraction for a total of 39 diffracting ROIs. Number of diffracting ROIs per grid ranged from 1 to 5 .

Random image acquisition or acquisition based on a predefined pattern would lead to a very time-consuming processing scheme, most images being irrelevant (acquired on grid bars, or absence of material). The selection of images being controlled by image processing, the amount of images and the time required to process a grid is reduced with our method. The images acquired at different magnifications are sufficiently representative to allow an efficient assessment of the sample by a specialist. Furthermore, the analysis of the results is assisted by the automatic statistic extraction made by the ANIMATED-TEM tools. A summary of the results is automatically generated to guide the user through the high amount of data collected by the system. Fig. S7 (Supplementary Materials) shows for each grid the processing time, the grid squares quality, the objects shapes (i.e. vesicles having round shapes, crystals with angular edges), the objects density (stacking), the average diameter of non-stacked object (single sheet crystal, being usually the most valuable crystal form for successful 3D reconstruction), and the ratio between diffracting/non-diffracting ROIs. This synthetic overview can also be displayed in a more detailed manner. When the grids of interest are identified, the user can navigate through the micrographs using an interactive interface that can be controlled solely with the computer's keyboard arrows for fast screening (See Fig. S8 and S9, Supplementary Materials).

\subsubsection{OmpF}

The bacterial porin $\mathrm{OmpF}$ is one of the first channel proteins extensively studied structurally, biochemically and functionally (Rosenbusch et al. 1990). Its reconstitution in regular arrays has been well studied (Holzenburg at al., 1989; Engel, et al., 1992; Rémigy et al., 2003; Signorell et al., 2007) and has been used to baseline our system (Fig. 6). The big objects resulting from OmpF crystallization were detected by automatic segmentation (Fig. 6B), and the multiple stacking detection was successfully applied (Fig. 6C). After the thinnest layer region selection (Fig. 6D), a high magnification micrograph was acquired (Fig. 6E, ROI n.1) and after Fourier transformation the diffraction spots revealed the presence of 2D crystals (Fig. 6F). Due to multiple stacking of crystals lattices depicted in Fig. 6E the power spectra contains numerous observable Friedel mates.

\subsubsection{HasA-HasR-heme complex}

The Has system, expressed when bacteria meet an iron deprived environment, allows the extracellular secretion of a protein with a high affinity for heme, called hemophore HasA (Benevides-Matos and Biville, 2010). HasA captures heme and returns it to an outer membrane protein HasR, its hemophore-specific receptor. HasA complexed with heme (holoHasA) as well as without (apoHasA) forms a tight complex with HasR. X-ray structure of HasA-HasR-heme complex was recently published (Krieg et al., 2009), but the 2D crystallization of the complex has not been performed yet. Therefore, we have used the HasA-HasR-heme to measure the screening performance of our system. Figure 7 shows typical results obtained with this membrane complex. Low magnification image (Fig. 7A) shows the automatic selection of 3 squares of good quality. These selected regions are ranked and images at medium magnification 
are taken at the corresponding positions, revealing after segmentation smaller objects than with OmpF (Fig. 7B). Stacking analysis identified numerous "single stacks" of small, round shaped objects (in red), corresponding to vesicles or small tubes. One-stacked membranes were targeted (Fig. 7D) for image acquisition at high magnification (Fig. 7E, ROI n.1) and the Fourier transform revealed multiple Friedel mates (Fig. 7F) due to the presence of a single, collapsed tube (Fig. 7E).

The wealth of data collected during OmpF and HasA-HasR-heme screens emphasized the need to develop a tool to gather the information generated at each step. This database should allow for 2D crystallization experiments to be setup (crystallization parameters: buffers and ternary mixtures composition, temperature profile etc...) for every common methods (dialysis, biobeads, monolayer and cyclodextrin) and results should be stored after screenings (electron microscopy parameters, micrographs, crystals quality, etc.). Also ternary mixture preparation protocols as well as cyclodextrin crystallization experiment protocols could be generated within this tool and directly exported into the ternary mixture and the cyclodextrin robots respectively and in return the experimental data can be recorded automatically in this laboratory information management system.

\section{Conclusions}

Although recent works give evidences that 2D crystallography is capable of achieving atomic resolution of membrane proteins in their native lipid bilayer environment, finding suitable crystallization conditions is a long process. After membrane protein purification and detergent concentration evaluation using the DropBox (Kaufmann 2006), this process can be divided in 5 steps, which are performed in parallel for a high number of samples: (i) protein-detergent-lipid ternary mixture preparation in various buffers with the addition of salts and ligands, (ii) crystallization by detergent neutralization at the suitable rate independently from the detergent nature (high or low CMC, harsh or mild detergent), (iii) staining of the crystals on grids suitable for TEM analysis, (iv) loading of the grids into the microscope, and (v) acquisition, analysis and classification of micrographs. In our tool-chain these steps are automatically performed by: (i) a ternary mixture robot, (ii) the 2DX robot based on detergent neutralization using the cyclodextrin method, (iii) the staining robot, (iv) the Carousel and the Autoloader, and (v) a Tecnai-12 controlled by ANIMATED-TEM. 2 proteins were selected to test our tool-chain. For the first membrane protein, OmpF, the system has detected crystals when known crystallization conditions were used. The crystallization conditions were not known for the second membrane protein, HasA-HasR-heme complex. Nevertheless the system could identify automatically successful conditions within the parameter space that has been chosen.

The process described in this paper was used to screen large numbers of $2 \mathrm{D}$ crystallization trials rapidly and automatically, enabling the biologist to focus on optimization of sample preparation. The results can be reviewed via a powerful navigation interface and stored in a laboratory information management system specifically developed for the HT3DEM (paper in preparation). The tool-chain presented in this paper could also be used to screen automatically single particles, cells fractionation aliquots and other non-biological samples at room temperature by developing specific segmentation algorithms in MATLAB.

Intensive efforts are currently undertaken to develop image processing packages to extract the structural information from the 2D crystals to the atomic level (2DX (Gipson et al., 2007), IPLT 
(Philippsen et al., 2007)). Such developments are solely driven by the analysis of data sets from new membrane proteins. High-throughput screening will help to gain insights in 2D crystallization faster, and we can anticipate an increase of atomic structures of membrane proteins determined by electron crystallography in a near future.

\section{Acknowledgments}

We are grateful to Jeroen van der Water, Roland Jonkers, Robert Wyss, Robert Haering and Raymond Strittmater for the Autoloader and the Carousel design, building and implementation, and Bart van der Schoot and Marcos Sancho for their technical support for the pipettors. Laurent Naris, Yoan Meret, Ibrahim Mansour and Sébastien Lehmann are acknowledged for their support during software development. We are grateful to Frédéric Huché and Philippe Delepelaire (Institut Pasteur, Paris) for providing HasA-HasR-heme complex and Kitaru Suda for OmpF purification. This work was supported by the HT3DEM project granted by European Union (project n. LSHG-CT-2005-018811; http://ht3dem.org), the University of Basel and the Maurice E. Müller Foundation of Switzerland.

\section{References}

Aebi, U., Pollard, T.D. 1987. A glow discharge unit to render electron microscope grids and other surfaces hydrophilic. J. Electron Microsc. Tech. 7, 29-33.

Al-Amoudi, A., Chang, J.J., Leforestier, A., MacDowall, A., Salamin, L.M., Norlén, L.P., Richter, K., Blanc, N.S., Studer, D., Dubochet, J. 2004, Cryo-electron microscopy of vitreous sections. EMBO J. 23, 3583-3588.

Benevides-Matos, N., Biville, F. 2010. The Hem and Has haem uptake systems in Serratia marcescens. Microbiology 156, 1749-1757.

Caillet-Saguy, C., Piccioli, M., Turano, P., Izadi-Pruneyre, N., Delepierre, M., Bertini, I., Lecroisey, A. 2009. Mapping the interaction between the hemophore HasA and its outer membrane receptor HasR using CRINEPT-TROSY NMR spectroscopy. J Am Chem Soc. 131, 1736-1744.

Carragher, B., Kisseber, N., Kriegman, D., Milligan, R. A., Potter, C. S., Pulokas, J., Reilein, A. 2000. Leginon: An Automated System for Acquisition of Images from Vitreous Ice Specimens. J. Struct. Biol. 132, 33-45.

Cheng, A., Leung, A., Fellmann, D., Quispe, J., Suloway, C., Pulokas, J., Priyanka, D. A., Lam, J. S., Carragher, B., Potter, C. S., 2007. Toward automated screening of two-dimensional crystals. J. Struct. Biol. 160, 324-331.

Cong, Y., Baker, M.L., Jakana, J., Woolford, D., Miller, E.J., Reissmann, S., Kumar, R.N., Redding-Johanson, A.M., Batth., T.S., Mukhopadhyay, A., Ludtke, S.J., Frydman, J., Chiu, W. 2010. 4.0-A resolution cryo-EM structure of the mammalian chaperonin TriC/CCT reveals its unique subunit arrangement. Proc Natl Acad Sc. 107, 4967-4972. 
Coudray, N., Buessler, J.-L., Kihl, H., Urban, J.-P. 2007. Automated image analysis for electron microscopy specimen assessment. 15th EUropean SIgnal Processing COnference (EUSIPCO 2007), PTETiS Poznan, Poland. 120-124.

Dubochet, J., McDowall, A.W. 1981. Vitrification of pure water for electron microscopy. J Microsc 124: RP3-RP4

Engel, A., Hoenger, A., Hefti, A., Henn, C., Ford, R.C., Kistler, J., Zulauf, M. J. 1992. Assembly of 2-D membrane protein crystals: dynamics, crystal order, and fidelity of structure analysis by electron microscopy. J. Struct. Biol. 109, 219-234.

Fellmann, D., Pulokas, J., Milligan, R. A., Carragher, B., Potter, C. S. 2002. A relational database for cryoEM: experience at one year and 50000 images. J. Struct. Biol. 137, 273-282.

Fujiyoshi, Y., Unwin N. 2008. Electron crystallography of proteins in membranes. Curr Opin Struct Biol. 18, 587-592.

Gipson, B., Zeng, X., Zhang, Z. Y., Stahlberg, H. 2007. 2dx- User-friendly image processing for 2D crystals. J. Struct. Biol. 157, 64-72.

Gonen, T., Sliz, P., Kistler, J., Cheng, Y., Walz, T. 2004. Aquaporin-0 membrane junctions reveal the structure of a closed water pore. Nature. 429, 193-197.

Gonen, T., Cheng, Y., Sliz, P., Hiroaki, Y., Fujiyoshi, Y., Harrison, S.C., Walz, T. 2005. Lipidprotein interactions in double-layered two-dimensional crystals of aquaporin-0. Nature. 438, 633-638.

Harris, J.R. 1997. Negative staining and cryoelectron microscopy BIOS scientific publishers.

Hasler, L., Heymann, J.B., Engel, A., Kistler, J. and Walz, T. 1998. 2D Crystallization of Membrane Proteins: Rationales and Examples. J. Struct. Biol. 121, 162-171.

Hayat, M.A., Miller, S.E. 1990. Negative Staining. McGraw-Hill Publishing Co.

Hite, R., Raunser, S., Walz K. 2007. Revival of electron crystallography. Current Opinion in Structural Biology. 17, 389-395.

Holm, P.J., Bhakat, P., Jegerschold, C., Gyobu, N., Mitsuoka, K., Fujiyoshi, Y., Morgenstern, R., Herbert, H. 2006. Structural basis for detoxification and oxidative stress protection in membranes. J. Mol. Biol. 360, 934-945.

Holzenburg, A., Engel, A., Kessler, R., Manz, H.J., Lustig, A., and Aebi, U. Rapid isolation of OmpF porin-LPS complexes suitable for structure-function studies. (1989) Biochemistry 28, 4187-4193.

Iacovache, I., Biasini, M., Kowal, J., Kukulski, W., Chami, M., van der Goot, F.G., Engel, A., Rémigy, H-W. 2010. The 2DX robot: A membrane protein 2D crystallization Swiss Army knife. J. Struct. Biol. 169, 370-378. 
Jap, B.K., Zulauf, M., Scheybani, T., Hefti, A., Baumeister, W., Aebi, U., Engel, A. 1992. 2D crystallization: from art to science. Ultramicroscopy. 46, 45-84.

Karathanou, A., Coudray, N., Hermann, G., Buessler, J.-L., Urban, J.-P. 2010. Automatic TEM image analysis of membranes for 2D crystal detection. Springer AEMB, Advances in Computational Biology. vol. 680, H. R. Arabnia, Ed.

Kaufmann, T.C., Engel, A., Rémigy, H-W. 2006. A novel method for detergent concentration determination. Biophys. J. 90, 310-317.

Krieg, S., Huché, F., Diederichs, K., Izadi-Pruneyre, N., Lecroisey, A., Wandersman, C., Delepelaire, P., Welte, W. 2009. Heme uptake across the outer membrane as revealed by crystal structures of the receptor-hemophore complex. Proc Natl Acad Sc. 106, 1045-1050.

Lefmann, J., Morrison, R., Subramaniam, S. 2007. Automated 100-position specimen loader and image acquisition sytem for transmission electron microscopy. J. Struct. Biol. 158, 318-326.

Mallick, S.P., Carragher, B.O., Potter, C.S., Kriegman, D.J. 2005. ACE: Automated CTF Estimation. Ultramicroscopy. 104, 8-29.

Morgenstern, R. 2005. Microsomal Glutathione Transferase 1.Methods in Enzymology. 401, 136-146.

Nakata, K., Inoue, Y. 1998. An automatic Bio-Beads-supplying device for two dimensional crystallization of membrane proteins. J. Appl. Crystallogr. 31, 644-645.

Nickell, S., Förster, F., Linaroudis, A., Net, W.D., Beck, F., Hegerl, R., Baumeister, W., Plitzko, J.M. 2005. TOM software toolbox: acquisition and analysis for electron tomography. J. Struct. Biol. 149, 227-234.

Philippsen, A. 2007. Collaborative EM image processing with the IPLT image processing library and toolbox. J. Struct. Biol. 157, 28-37.

Potter, C., Pulokas, J., Smith, P., Suloway, C., Carragher, B. 2004. Robotic grid loading system for a transmission electron microscope. 146, 431-440.

Rémigy, H-W., Caujolle-Bert, D., Suda, K., Schenk, A., Chami, M., Engel, A. 2003. Membrane protein reconstitution and crystallization by controlled dilution. FEBS Lett. 555, 160-169.

Rigaud, J.L., Mosser, G., Lacapere, J-J., Olofsson, A., Levy, D., Ranck, J-L. 1997. Bio-Beads: An efficient Strategy for Two-Dimensional Crystallization of Membrane Proteins. J. Struct. Biol. $118,226-235$.

Rigaud, J.L., Chami, M., Lambert, O., Levy, D., Ranck, J.L. 2000. Use of detergents in twodimensional crystallization of membrane proteins. Biochimica et Biophysica Acta. 1508, 112128.

Ringler, P., Heymann, B.J., Engel, A., 2000. Two-dimensional crystallization of membrane proteins. In: Baldwin, S.A. (Ed.), Membrane Transport. Oxford University Press, Oxford, pp. 229-268. 
Rosenbusch, J.P. 1990. Structural and functional properties of porin channels in E. coli outer membranes. Experientia. 46, 167-173.

Signorell, G.A., kaufmann, T.C., Kukulsi, W., Engel, A., Rémigy, H-W. 2007. Controlled 2D crystallization of membrane proteins using methyl-beta-cyclodextrin. J. Struct. Biol. 157, 321328.

Suloway, C., Pulokas, J., Fellman, D., Cheng, A., Guerra, F., Quispe, J., Stagg, S., Potter, C. S., Carragher, B. 2005. Automated molecular microscopy: The new Leginon system. J. Struct. Biol. $151,41-60$.

Tani, K., Mitsuma, T., Hiroaki, Y., Kamegawa, A., Nishikawa, K., Tanimura, Y., Fujiyoshi, Y. 2009. Mechanism of Aquaporin-4's Fast and Highly Selective Water Conduction and Proton Exclusion. J. Mol. Biol. 389, 694-706.

Unwin, N. 2005. Refined structure of the nicotinic acetylcholine receptor at $4 \AA$ resolution. J. Mol. Biol. 346, 967-989.

Vink, M., Derr, D. K., Love, J., Stokes, D. L., Ubarretxena-Belandia, I., 2007. A high-throughput strategy to screen 2D crystallization trials of membrane proteins. J. Struct. Biol. 160, 295-304. 
Table 1: Average-case running times for OmpF and HasA-HasR-heme 96 grids run (54 hours of automatic screening).

Figure 1: Automatic grid staining and screening. (A) Close up of the pipetting system preparing 4 EM grids at once (sample deposit, blotting, washing and staining); (B) 12 Grids are inserted in a Cassette; (C) 8 Cassettes are inserted in the Carousel; (D) The Carousel is attached to the Autoloader of the microscope; (E) FEI's Tecnai 12 equipped with the Autoloader and the Carousel. When less than 12 grids need to be screened, a Capsule hosting only one Cassette can also be docked into the Autoloader (Arrow).

Figure 2: Grid square quality assessment at low magnification. (A) Typical histograms of nonbroken (above) and broken (below) carbon film grid squares. (B) Outcome from the automatic grid square selection. Scale bar is $50 \mu \mathrm{m}$.

Figure 3: ROIs detection by image segmentation and labeling at medium magnification. (A) Initial medium magnification image. (B) Outcome of the image partition (blue lines). (C) Validation of the partition through merging of red lines. (D) Multi-stacking modeling (background in black, one-, two-, three, and more-than-three-stacked membranes in red, green, magenta, and blue respectively). Scale bar is $2 \mu \mathrm{m}$.

Figure 4: Assessment of crystallinity at high magnification. (A) Initial image. (B) Power spectra of (A). (C) Mean radial gray level profile $\mathrm{R}_{\mathrm{rc}}\left(\mathrm{C}_{\mathrm{ROI}}\right.$ Circle Radius) of the power spectra (B) measured over $\mathrm{C}_{\mathrm{ROI}}$ (Circle delimiting the Regions Of Interest) from 0 to $\pi$ (represented by the solid arc). (D) Identified diffraction peaks. Scale bar is $200 \mathrm{~nm}$.

Figure 5: Screening session scenario control Graphical User Interface (GUI).

Figure 6: Automatic screening of OmpF 2D crystals. (A) Grid square selection at low magnification, Scale bar $50 \mu \mathrm{m}$. (B) Linearity of the edges on the medium magnification image (linear edges in red, more circular edges in blue, others in black). (C) Stacking-level representation of the membranes (one-stacked regions in red, two-stacked regions in green, highly-stacked regions in purple). (D) Membrane image at medium magnification with the selection of 2 ROIs (red circles) based on the multi-resolution method, Scale bar $2 \mu \mathrm{m}$. (E) High magnification image, Scale bar $100 \mathrm{~nm}$. (F) Diffraction pattern of the crystal, identified diffraction spots being highlighted in red, blue or green (for spots with high, medium or low signal to noise ratio respectively).

Figure 7: Automatic screening of HasA-HasR-heme 2D crystals. (A) Grid square selection at low magnification, Scale bar $50 \mu \mathrm{m}$. (B) Detection of the edges on the medium magnification image. (C) Stacking-level representation of the membranes. (D) Membrane image at medium magnification with the selection of 4 ROIs, Scale bar B-D: $2 \mu \mathrm{m}$. (E) High magnification image of area 1 in D, Scale bar $100 \mathrm{~nm}$. (F) Diffraction pattern of the single collapsed crystalline tube in $\mathrm{E}$ and identified spots.

Table S1: Success rate comparison between automatic and manual selection.

Table S2: Microscope control scenario. 
Figure S3: (A) Ternary Mixture Robot. (B) Experiment design template. Constraints related to the equipments used (robot performances, recipient sizes, sample volumes, maximum final concentrations etc.) and the screening conditions (final protein/lipid/detergent/additive concentrations etc.) are entered in this interface. Calculations are automatically performed to check whether constraints and screening conditions are compatible. In that case, a mixing protocol will be generated from this interface. (C) Control interface used to load the mixing protocol in the robot and to start a run and to perform the initialization and the maintenance of the robot.

Figure S4: Staining Block. (A) Top view of the staining block. (B) Transparent view of the block, showing the magnets lying underneath the nickel grids. (C) Close up of the staining robot pipetting system. The pipets in the front are used to deposit sample, water for grid washing, and finally staining. The actuated pipets visible in the back are used for sample blotting, water elimination during washing, and staining blotting.

Figure S5: Staining Robot User Interface. Through this interface, the operator can control each staining parameter (sample incubation time, amount of blotting cycles, staining incubation time) but also all the actions needed for maintenance.

Figure S6: Interactive grid to screen selection.

Figure S7: Summary of experimental results. Processing time, grid squares quality, objects density, objects shape, average non-stacked objects diameter, and objects density are visible at a glance for all 96 grids.

Figure S8: Navigation interface to review results at low and medium magnifications. The area selected at low magnification (left pane) is shown at middle magnification (right pane).

Figure S9: Navigation interface to review results at medium and high magnification. The ROIs selected at middle magnification (right pane) are shown at high magnification (left pane). 

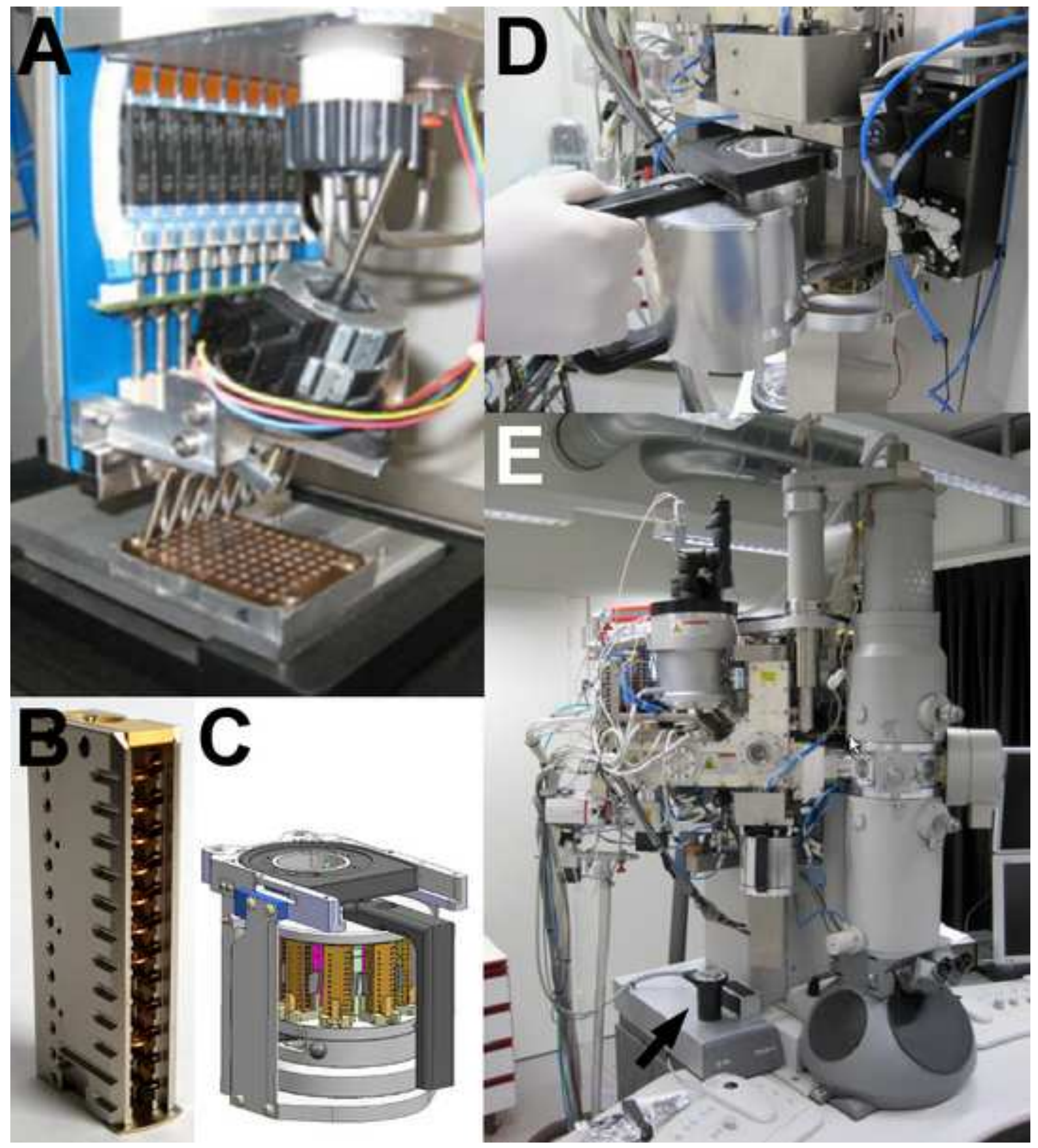

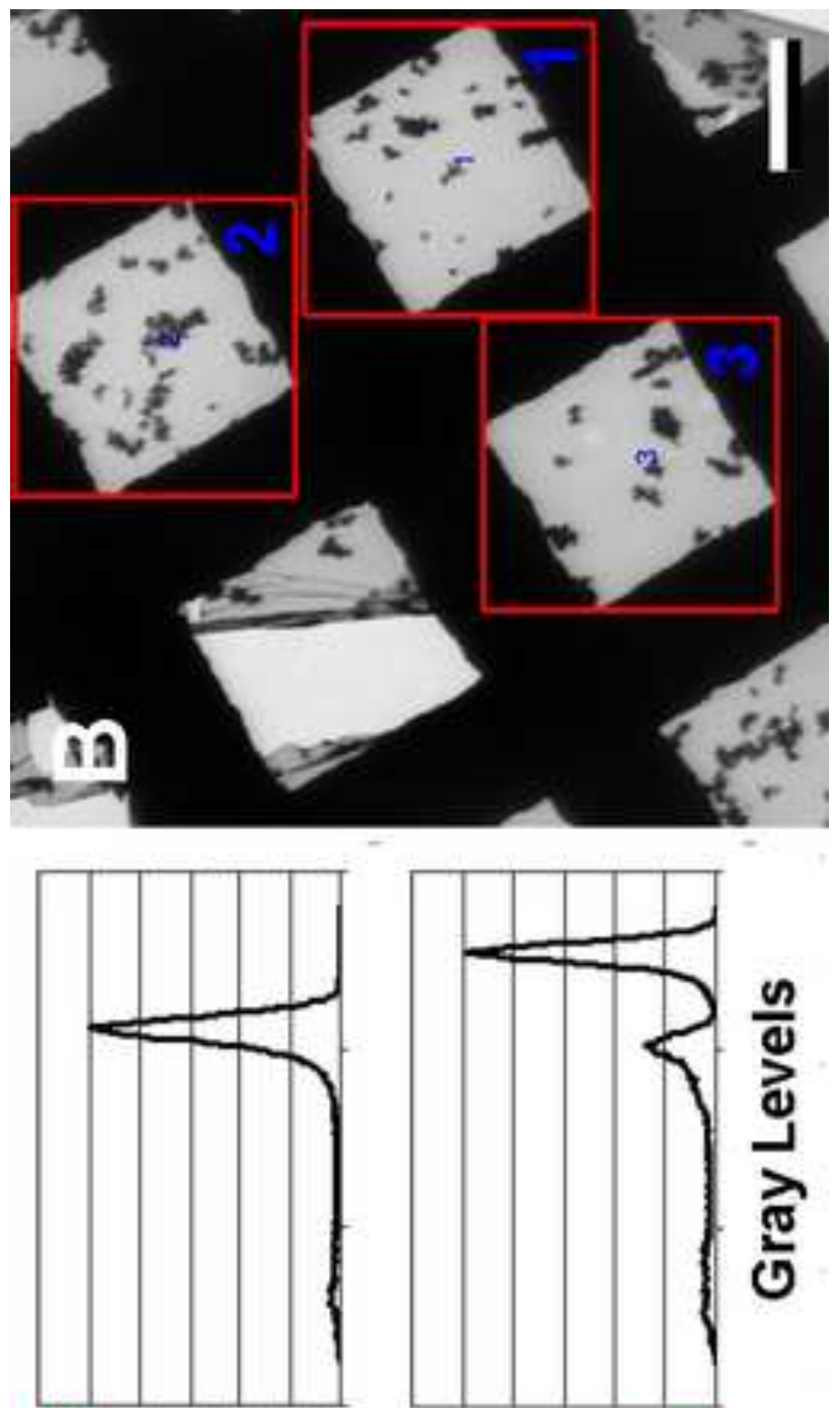

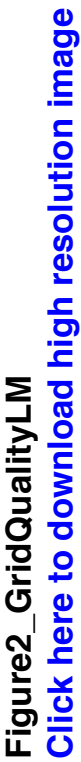

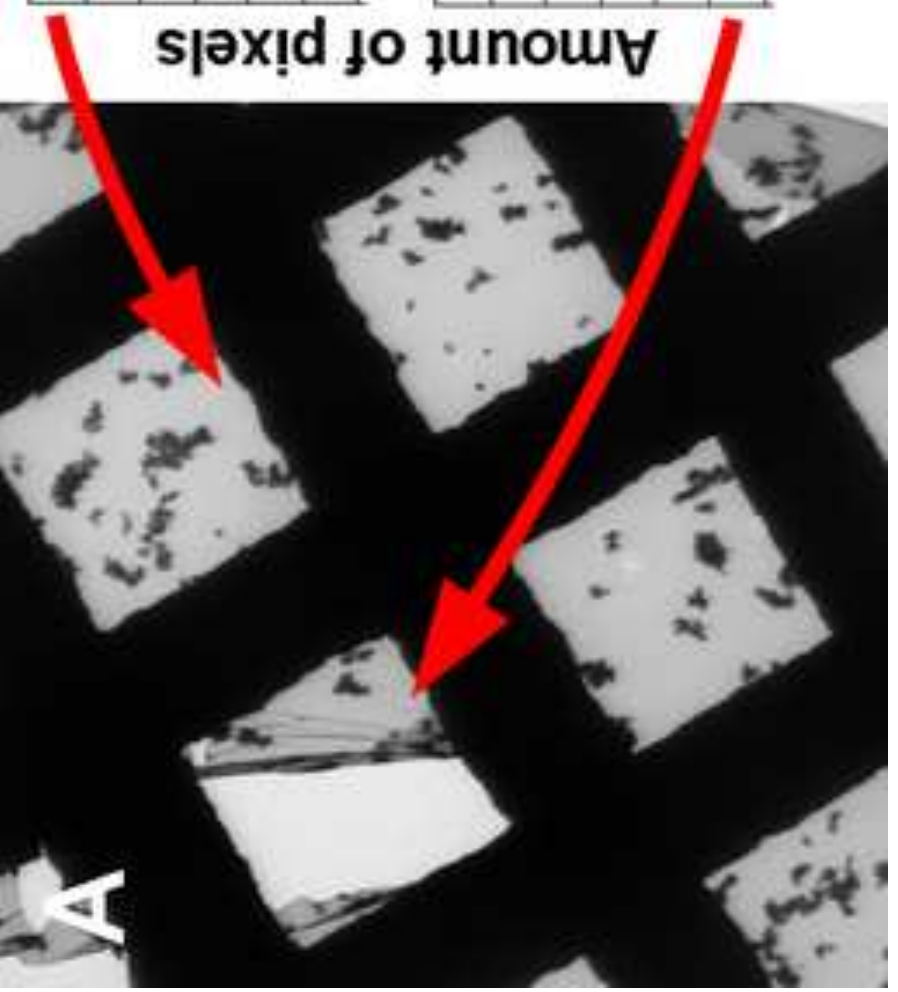



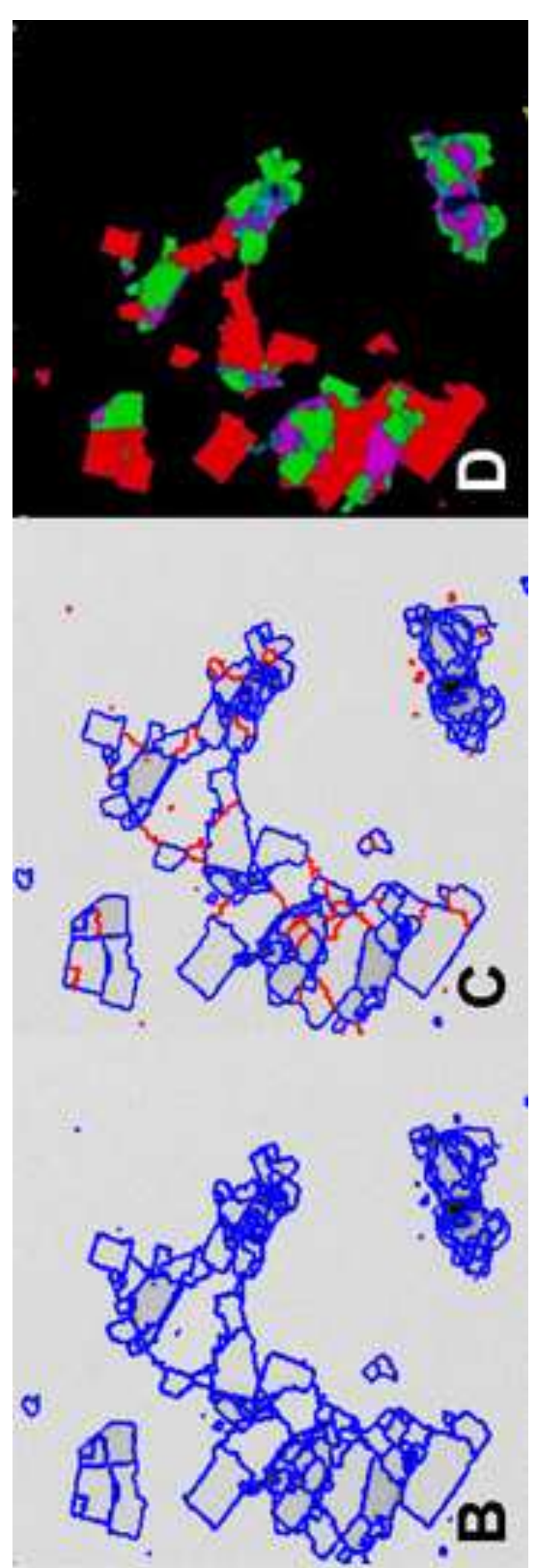

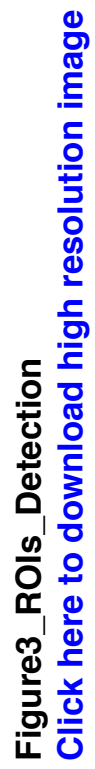

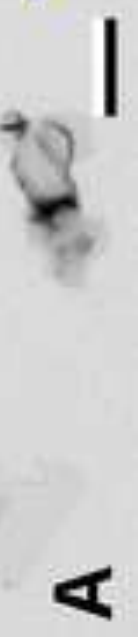



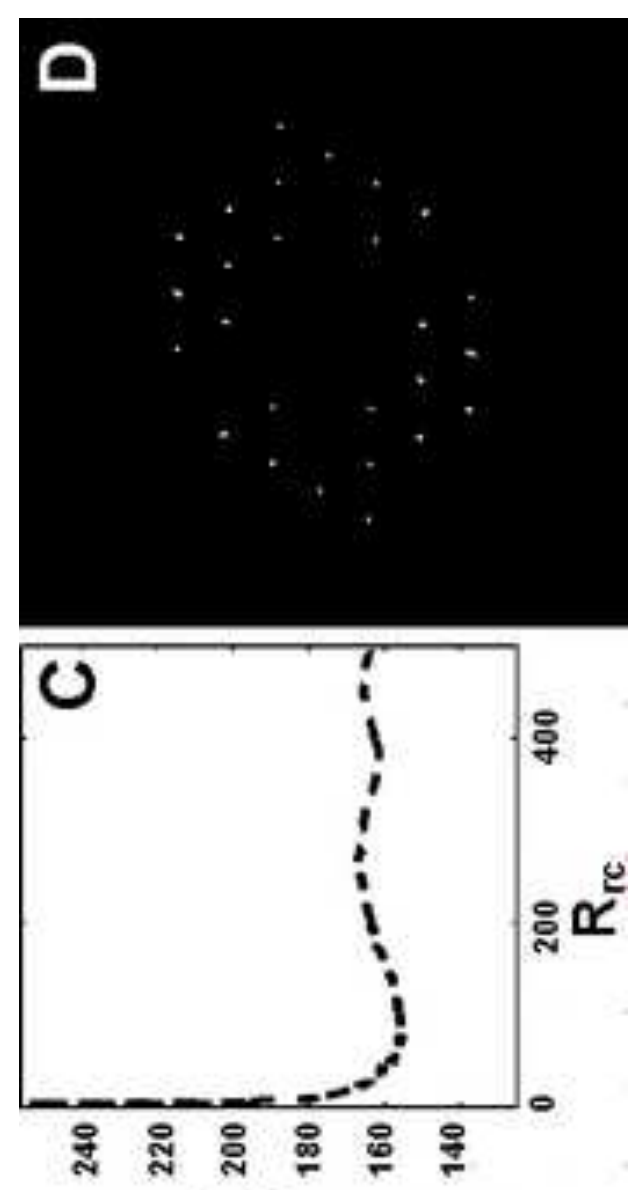

ןөле| Кел6 ןе!pes ueəW

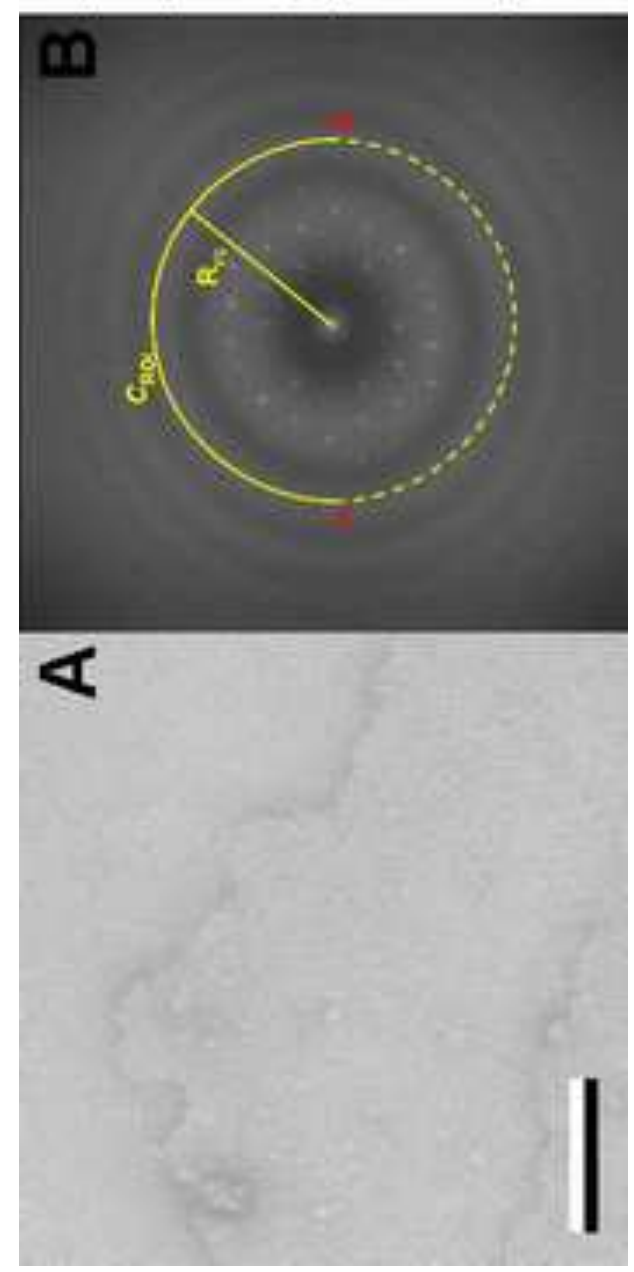

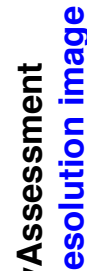

>

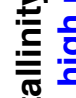
. 年 


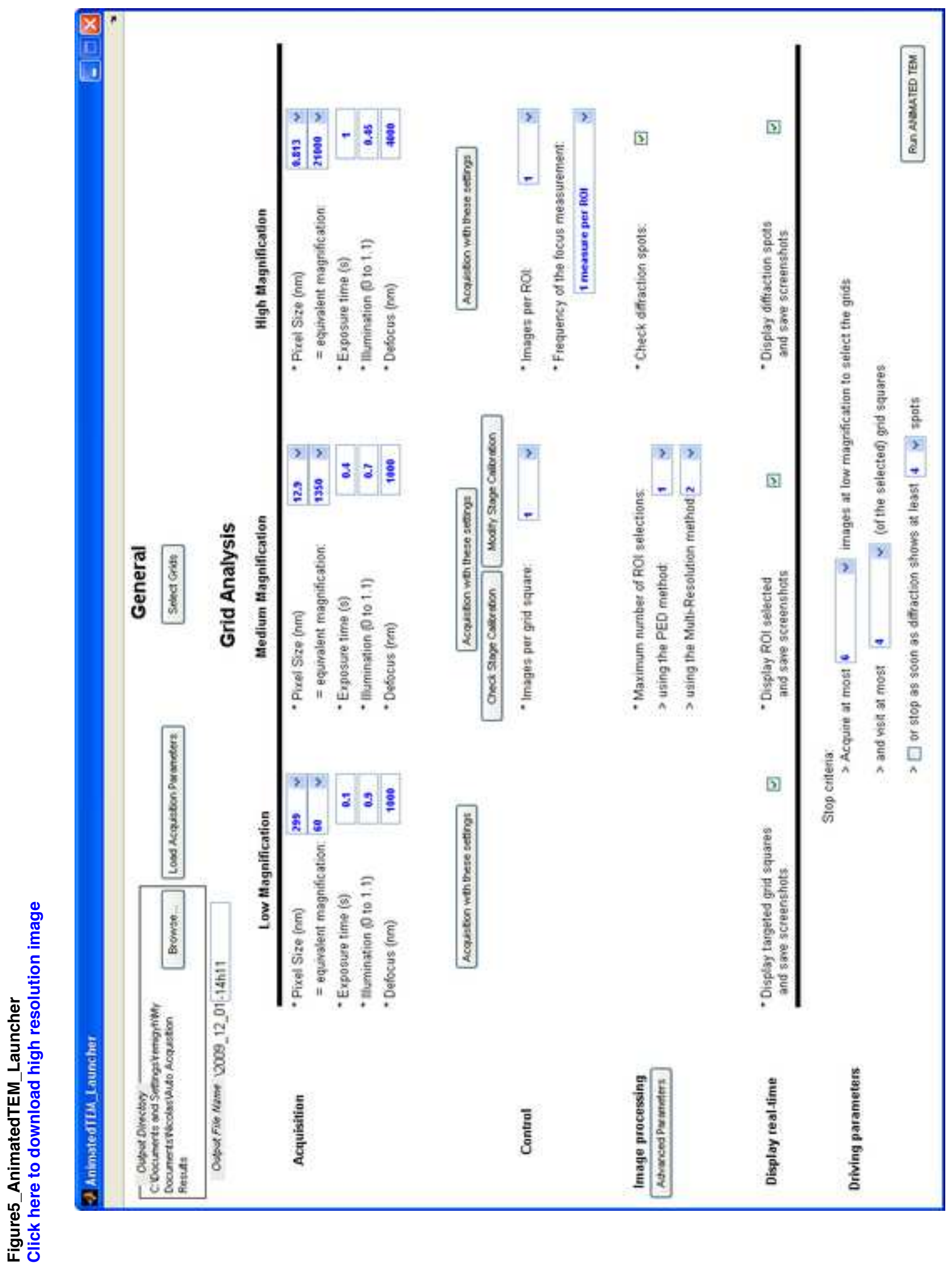




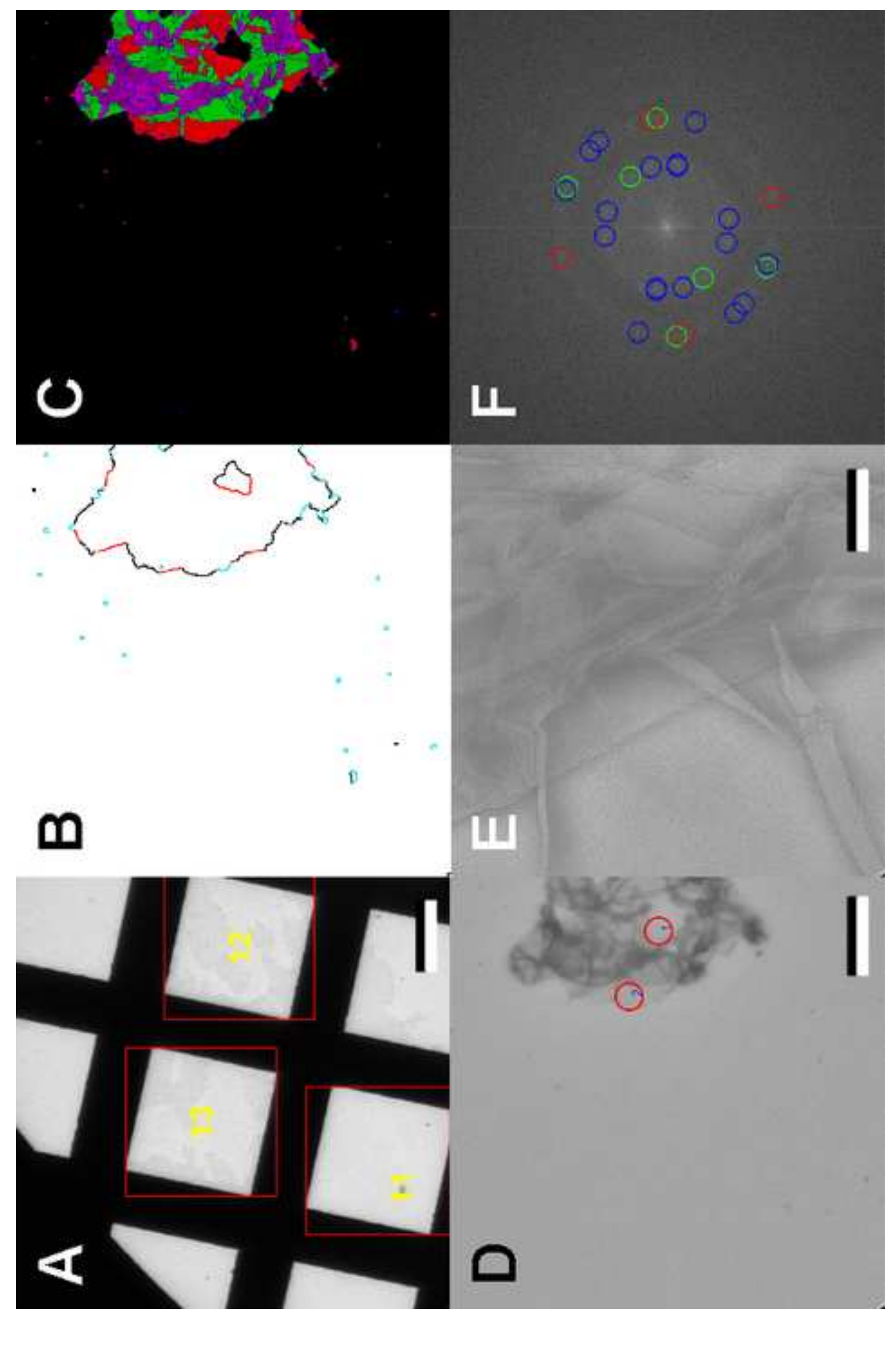




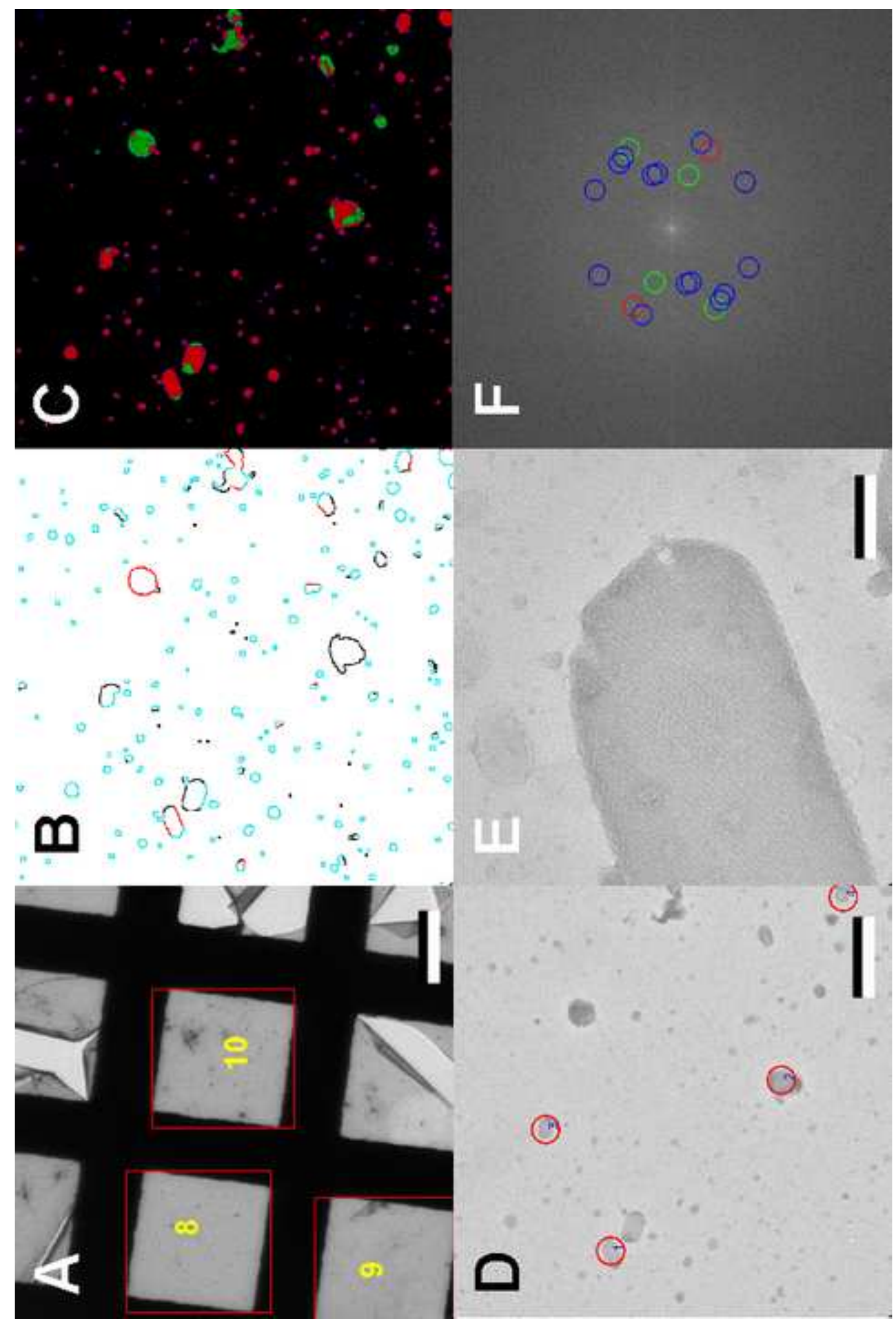

웡 5 


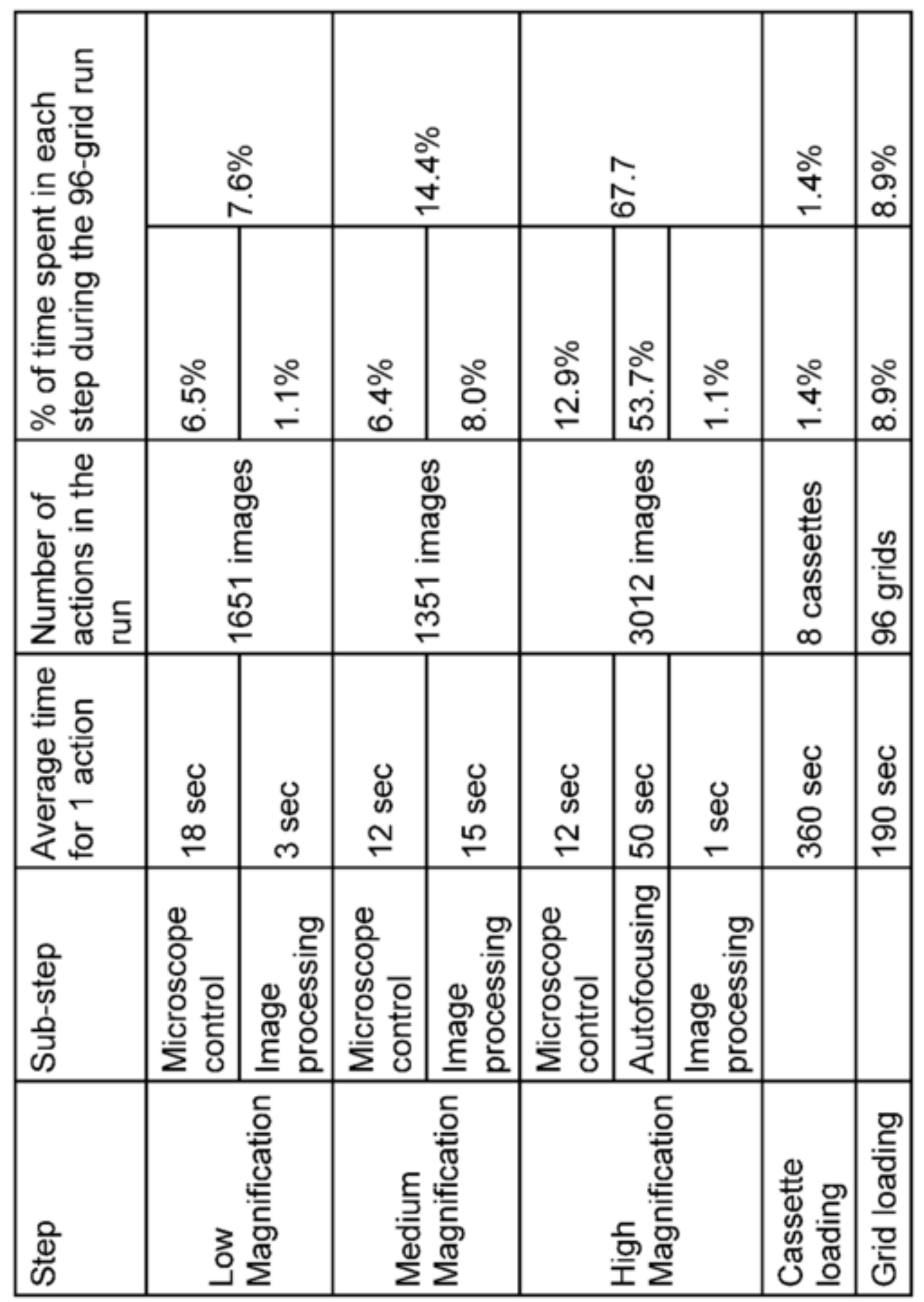

